Baltic Astronomy, vol. 6, 353, 1997.

\title{
THE VOLUME OF INFORMATION EXPECTED IN THE SPACE ASTROMETRY PROJECT STRUVE
}

\section{M. Kopylov and M.S. Chubey}

Pulkovo Observatory, Russian Academy of Sciences, St. Petersburg 196140, Russia

Received July 21, 1996.

Key words: astrometry - space vehicles - instrumentation

The data acquisition operational system in the space astrometry project STRUVE is being designed on the basis of the CCD-mosaic registration system. Two focal assemblies consist of $28 \mathrm{CCD}$-arrays, each of $800 \times 800$ pixels of $16 \mu \mathrm{m}$ size. Two Schmidt telescopes of aperture $40 \mathrm{~cm}$, focal length of $2.5 \mathrm{~m}$ and angular field of view of 2.41 square degrees will be used, and $5.1 \cdot 10^{6}$ pixels must be read every second of time. The dynamic range of the CCD-sensitivity is $2 \cdot 10^{5} e^{-}$. The full downlink output is $\approx 5 \mathrm{Mbaud}$. The margin magnitude on the limit range of sensitivity is $V \approx 22 \mathrm{mag}$ in the integral passband $\Delta \lambda \in(0.33-0.75) \mu \mathrm{m}$ and is near to $V=18 \mathrm{mag}$ with a signal-to-noise ratio $\approx 3$ in the passbands of the Vilnius sevencolor photometric system. The final databases are supposed to be:

1. The raw CCD-registration strip files: $\approx 50$ Tbyte.

2. The Reference Astrometric and Photometric Catalog database of $\approx 14$ Gbyte. The positional and parallax accuracy for all stars brighter $V=14 \mathrm{mag}$ must be better than 0.4 milliarcsecond, the photometric accuracy in all bands should be better than 4 millimagnitudes.

3. The Astronomical Image Data Base with the reduced CCD-data: $\approx 500$ Gbyte.

Thus, the STRUVE project is subject to the construction of fundamental coordinate system with proper motions linked to cosmological objects. The overview of faint stars is expected to be complete down to $V \approx 18 \mathrm{mag}$ in multicolor passbands and potentially down to $V \approx 21-22 \mathrm{mag}$ in the integral band. 